\title{
The Microanatomic Location of Metastatic Breast Cancer in Sentinel Lymph Nodes Predicts Nonsentinel Lymph Node Involvement
}

\author{
Carolien H. M. van Deurzen, MD, ${ }^{1}$ Cees A. Seldenrijk, MD, PhD, ${ }^{1}$ \\ Ron Koelemij, MD, PhD, ${ }^{2}$ Richard van Hillegersberg, $\mathrm{MD}, \mathrm{PhD},{ }^{3}$ \\ Monique G. G. Hobbelink, MD, ${ }^{4}$ and Paul J. van Diest, MD, $\mathrm{PhD}^{5}$
}

\author{
${ }^{1}$ Department of Pathology, St Antonius Hospital, Nieuwegein, The Netherlands \\ ${ }^{2}$ Department of Surgery, St Antonius Hospital, Nieuwegein, The Netherlands \\ ${ }^{3}$ Department of Surgery, University Medical Center Utrecht, Utrecht, The Netherlands \\ ${ }^{4}$ Department of Nuclear Medicine, University Medical Center Utrecht, Utrecht, The Netherlands \\ ${ }^{5}$ Department of Pathology, University Medical Center Utrecht, 85500, Utrecht, GA 3508, The Netherlands
}

\begin{abstract}
Background: The majority of sentinel node (SN) positive breast cancer patients do not have additional non-SN involvement and may not benefit from axillary lymph node dissection (ALND). Previous studies in melanoma have suggested that microanatomic localization of SN metastases may predict non-SN involvement. The present study was designed to assess whether these criteria might also be used to be more restrictive in selecting breast cancer patients who would benefit from an ALND.

Methods: A consecutive series of 357 patients with invasive breast cancer and a tumorpositive axillary SN, followed by an ALND, was reviewed. Microanatomic SN tumor features (subcapsular, combined subcapsular and parenchymal, parenchymal, extensive localization, multifocality, and the penetrative depth from the SN capsule) were evaluated for their predictive value for non-SN involvement.

Results: Non-SN metastases were found in 136/357 cases (38\%). Microanatomic location and penetrative depth of SN metastases were significant predictors for non-SN involvement $(<0.001)$; limited penetrative depth was associated with a low frequency of non-SN involvement with a minimal of $10 \%$.

Conclusions: Microanatomic location and penetrative depth of breast cancer SN metastases predict non-SN involvement. However, based on these features no subgroup of patients could be selected with less than $10 \%$ non-SN involvement.
\end{abstract}

Key Words: Breast cancer-Sentinel node-Axillary lymph node metastases-Morphometry.

Axillary nodal status is among the most important prognostic factors in breast cancer patients. SN biopsy with an intensive pathological assessment of selectively removed lymph nodes is currently a highly accurate, minimally invasive technique to assess no-

Published online February 6, 2008.

Address correspondence and reprint requests to: Paul J. van Diest, MD, PhD; E-mail: P.J.vanDiest@umcutrecht.nl

Published by Springer Science+Business Media, LLC $\odot$ The Author(s) 2008 dal status. ${ }^{1,2}$ It reduces the morbidity of breast cancer surgery by avoiding unnecessary axillary lymph node dissection (ALND) in patients with negative sentinel nodes (SNs). ${ }^{3}$ The optimal treatment of patients with a positive $\mathrm{SN}$ is however less clear. The few SNs can be cost-effectively analyzed by multiple-level evaluation and immunohistochemistry (IHC) which increases the likelihood of detecting small metastases. ${ }^{4}$ The decision to proceed with an ALND in patients with macrometastatic SN involvement does not pose 
a major clinical dilemma. The need for routine ALND in patients with minimal SN involvement however continues to be debated since only a minority of these patients $(10-15 \%)$ show non-SN involvement. ${ }^{5}$ Predicting the chance of involvement of the non-SN would facilitate the selection of patients with a potential therapeutic benefit of ALND.

Several features of the primary tumor and the involved SNs have been investigated as potential predictors for non-SN involvement. Primary tumor size, palpability, presence of peritumoral lymphovascular invasion, number of tumor-involved SNs, size of the SN metastases, and extracapsular extension (ECE) correlate with non-SN status. ${ }^{6-10}$ However, none of these factors are sensitive and reproducible enough to reliably identify a subgroup of patients who might be spared ALND.

Several melanoma studies have reported that the microanatomic pattern of $\mathrm{SN}$ involvement and the penetrative depth (defined as the maximum distance of cancer cells from the inner margin of the SN capsule) predict non-SN involvement. ${ }^{11-14}$ In breast cancer, this has been studied less extensively. ${ }^{15} \mathrm{We}$ therefore set out to study the predictive value of microanatomic location and penetrative depth of SN metastatic deposits for non-SN metastases, accurately assessed by morphometry, in a large series of $\mathrm{SN}$-positive breast cancer patients.

\section{PATIENTS AND METHODS}

\section{Patients}

A retrospective database was analyzed, including patients with invasive breast cancer and a tumor positive axillary SN followed by ALND, treated at the University Medical Center Utrecht or the St Antonius Hospital in Nieuwegein from January 2000 to May $2007(n=357)$, including patients from our previous study. ${ }^{16}$ Exclusion criteria were multicentric tumors, neoadjuvant chemotherapy, and a total of fewer than six lymph nodes examined.

\section{SN Biopsy Technique}

The technical aspects used for the SN procedure are described in detail elsewhere. ${ }^{16}$ Briefly, before surgery, SN identification was performed by peritumoral injection of $120 \mathrm{MBq}{ }^{99 \mathrm{~m}} \mathrm{Tc}-$ Nanocolloid (Amersham Cygne, Eindhoven, The Netherlands) in a maximal volume of $0.5 \mathrm{~mL}$. Dynamic and static scintigraphic images were subsequently obtained. On the same day, immediately preoperatively $0.5 \mathrm{~mL}$ Patent blue dye (Guerbet, Aulnay-sous-Bois, France) was injected intradermally and intra/peritumorally. The SN was identified after careful dissection of blue lymphatic channels and detection of radioactivity with a handheld gamma ray detection probe. Palpation of the open axilla was performed to detect enlarged non-SN metastases.

\section{Histopathological Evaluation}

The SNs were processed according to the protocols described previously. ${ }^{16-18}$ SNs were lamellated according to their size, fixed in neutral buffered formaldehyde, and completely embedded. Step sections $5 \mu \mathrm{m}$ thick were cut at five levels with $250 \mu \mathrm{m}$ intervals for staining with haematoxylin and eosin (H\&E). In the absence of apparent metastases by H\&E examination, immunohistochemistry was performed with CAM 5.2 (Beckton Dickinson, Franklin Lakes, New Jersey, USA) or CK AE1/3 (Dako, Glostrup, Denmark) at each level. All non-SNs were identified visually or by palpation, dissected, processed routinely, and examined at one level with $\mathrm{H} \& \mathrm{E}$ staining. All SNs and non-SNs were examined initially by multiple pathologists at the two institutions, reviewed histologically, and reclassified according to the current $6^{\text {th }}$ edition of the American Joint Committee on Cancer (AJCC) staging system by one observer $(\mathrm{CHMvD})$. All cases were evaluated without knowledge of non-SN involvement.

\section{Clinicopathological Features}

Clinicopathological features recorded included age, pT (TNM system of the AJCC), histological subtype (according to the WHO), histological grade (defined according to the Nottingham modified BloomRichardson score based on the percentage of tubule formation, nuclear pleomorphism and mitotic activity), mitotic activity index (MAI), ${ }^{19}$ steroid receptor and HER-2/neu status (not routinely determined before 2005).

\section{SN and Non-SN Characteristics}

SN characteristics included metastatic size according to the $6^{\text {th }}$ edition of the AJCC staging system [isolated tumor cells (ITC) $(\leq 0.2 \mathrm{~mm})$, micrometastases ( $>0.2 \mathrm{~mm}$ and $\leq 2 \mathrm{~mm}$ ), macrometastases $(>2 \mathrm{~mm})$ ], the number of SNs, ECE, maximal diameter of the largest metastases, microanatomic location of the metastatic deposit, and the penetrative 


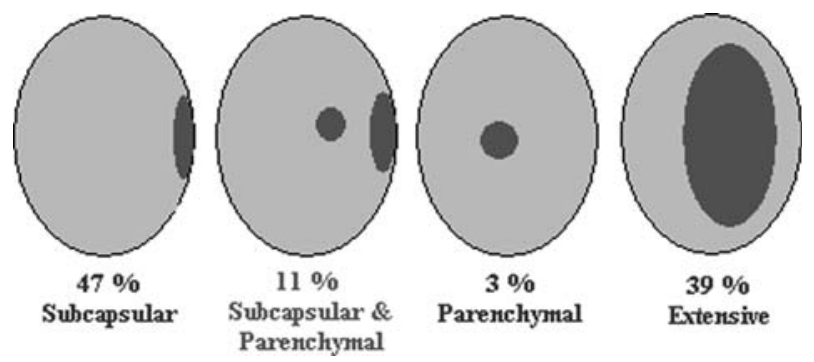

FIG. 1. Pattern of distribution of metastatic breast cancer deposits in SNs.

depth. If multiple but distinct deposits were identified in the same SN, the largest metastasis was recorded. If single tumor cells, cluster of nests were continuous, or separated by a few cells distance, they were measured as one focus. ${ }^{20}$ If more than one $\mathrm{SN}$ was involved in an individual patient, the most extensive and/or deepest metastatic deposit was recorded. In case both axillary and internal mammary SNs were involved, the features were measured in the axillary SN.

The microanatomic location of metastatic deposits within each SN was classified as subcapsular, combined subcapsular and parenchymal, parenchymal or extensive. Extensive SN involvement, as defined in the study of Ruiter et al., ${ }^{21}$ was a deposit $>5 \mathrm{~mm}$ in diameter (Fig. 1). The centripetal depth was, according to Starz et al., ${ }^{11}$ defined as the maximal depth at which tumor cells have infiltrated the $\mathrm{SN}$, as measured from the inner margin of the capsule (Fig. 2), further denoted tumor penetrative depth according to the proposal of Scolyer et al. ${ }^{12}$ Multifocality was defined as two or more separated metastatic deposits at some distance from each other. All measurements were calculated microscopically in the plane of the tissue sections using interactive video morphometry systems (QPRODIT, Leica, Cambridge, UK or Research Video Assistant, Baarn, The Netherlands).

Non-SN characteristics included the total number of non-SNs, maximal tumor diameter, AJCC classification, and ECE. If more than one non-SN was involved, the largest diameter was recorded.

\section{Statistical Analysis}

Statistical analysis was performed using SPSS 13.0 for Windows. Patients were divided into groups with and without non-SN involvement. The Pearson chisquare test was used to determine the relationship between categorical variables (histological type and grade, steroid receptor and HER-2/neu status, number of SNs, AJCC classification, ECE) on the one

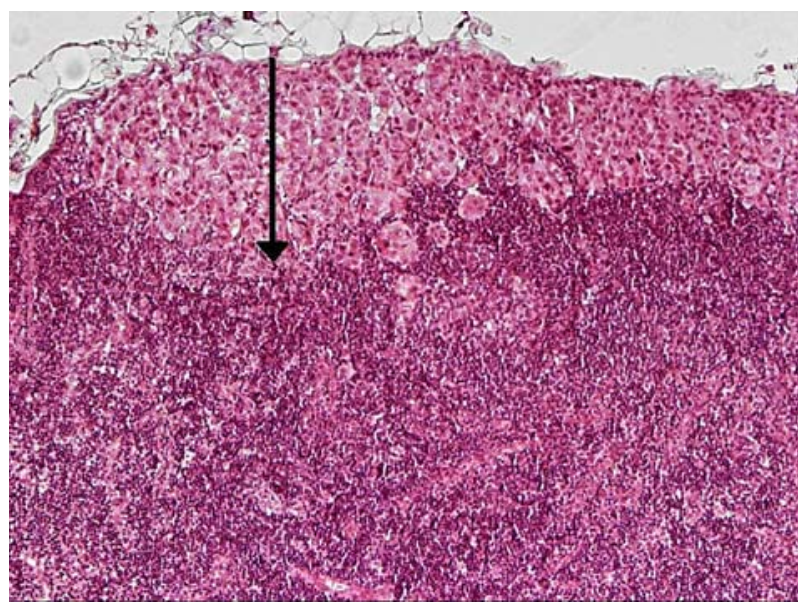

FIG. 2. The tumor penetrative depth of metastases was defined as the maximal distance of breast cancer cells from the inner margin of the $\mathrm{SN}$ capsule (arrow) $(\mathrm{H} \& \mathrm{E}$, original magnification $\times 10)$.

hand and non-SN status on the other. Continuous data (age, diameter primary tumor, MAI, SN tumor diameter) were analyzed using the Mann-Whitney $U$ test. $P$-values $<0.05$ were considered significant. All relevant variables that were associated with the presence of positive non-SNs were included in a multivariate logistic regression model. SN metastatic characteristics (diameter and penetrative depth) were further compared by receiver operating characteristic (ROC) analysis, calculating the area under curve (AUC) as a measure of discriminative value.

\section{RESULTS}

The median age was 53 years (range $22-86$ years) and the mean histological invasive tumor size was $2.4 \mathrm{~cm}$ (range $0.3-9.0 \mathrm{~cm}$ ). Overall, $571 \mathrm{SNs}$ were obtained (mean 1.6 SN per patient) of the 357 patients with a positive SN as well as 4939 non-SNs (mean 14 per patient). The metastatic deposits were subcapsular in 167 patients (47\%), combined subcapsular and parenchymal in 40 patients $(11 \%)$, parenchymal in 11 patients $(3 \%)$, and extensive in 139 patients (39\%) (Fig. 1). Of the 357 patients, 24 (7\%) patients had ITC only in the SN, whereas 112 $(31 \%)$ had micrometastases and $221(62 \%)$ had macrometastases. Localisation of ITCs was subcapsular $(96 \%)$ or parenchymal $(4 \%)$. SN micrometastases were located subcapsularly $(81 \%)$, parenchymally $(5 \%)$ or had combined localisation $(13 \%)$. The overall prevalence of non-SN involvement was 38\% (136/357 patients). Other descriptive characteristics of the study population are listed in Tables 1 and 2. 
TABLE 1. Clinicopathological characteristics of 357 invasive breast cancer patients with a positive $S N$ and subsequent axillary lymph node dissection

\begin{tabular}{|c|c|c|}
\hline Feature & No. & $\%$ \\
\hline Mean age (range) & 54 y $(22-86)$ & \\
\hline \multicolumn{3}{|l|}{ Primary tumor } \\
\hline Mean tumor size (range) & $2.4 \mathrm{~cm}(0.3-9.0)$ & \\
\hline pT1 & 187 & 52 \\
\hline pT2 & 150 & 42 \\
\hline pT3 & 19 & 5 \\
\hline pTx & 1 & 0 \\
\hline \multicolumn{3}{|l|}{ Histological subtype } \\
\hline Invasive ductal cancer & 298 & 84 \\
\hline Invasive lobular cancer & 36 & 10 \\
\hline Others & 23 & 6 \\
\hline \multicolumn{3}{|l|}{ Histological grade (B\&R) } \\
\hline 1 & 74 & 21 \\
\hline 2 & 161 & 45 \\
\hline 3 & 122 & 34 \\
\hline MAI, mean $/ 2 \mathrm{~mm}^{2}$ (range) & $13(0-102)$ & \\
\hline \multicolumn{3}{|l|}{ Steroid receptor status } \\
\hline ER - positive $\dagger$ & 322 & 90 \\
\hline ER - negative & 34 & 10 \\
\hline ER - unknown & 1 & \\
\hline $\mathrm{PR}-$ positive $\dagger$ & 284 & 80 \\
\hline $\mathrm{PR}$ - negative & 70 & 20 \\
\hline PR - unknown & 3 & \\
\hline \multicolumn{3}{|l|}{ HER-2/neu status } \\
\hline Positive & 26 & 15 \\
\hline Negative & 150 & 85 \\
\hline Unknown & 181 & \\
\hline
\end{tabular}

$\dagger \geq 10 \%$ immunoreactive neoplastic cells.

TABLE 2. $S N$ and non-SN characteristics of 357 invasive breast cancer patients with a positive $S N$ and subsequent axillary lymph node dissection

\begin{tabular}{llr}
\hline Feature & No. & $\%$ \\
\hline SN & & \\
Total number of SNs & 571 & \\
Mean number of SNs & 1.6 & \\
Total number of positive SNs & 419 & \\
Mean diameter SN metastases & $4.7 \mathrm{~mm}$ & 7 \\
AJCC classification of SN metastatic size & & 31 \\
ITC & 24 & 62 \\
Micrometastasis & 112 & \\
Macrometastasis & 221 & 28 \\
Extracapsular extension & & \\
No & 257 & 37 \\
Yes & 100 & 11 \\
Microanatomic location & 167 & 39 \\
Subcapsular & 11 & \\
Parenchymal & 40 & \\
Combined & 139 & \\
$\quad$ Extensive & $2.9 \mathrm{~mm}$ & \\
Mean penetrative depth & & \\
Non-SNs & 4939 & \\
Total number of non-SNs & $14(5-38)$ & \\
Mean number of non-SNs (range) & 474 & \\
Total number of positive non-SNs & 4465 & \\
Total number of negative non-SNs & & \\
Size of non-SN metastases & 3 & \\
ITC & 33 & \\
Micrometastases & 100 & \\
Macrometastases & & \\
\hline
\end{tabular}

The following factors were significant predictors of non-SN metastases by univariate analysis: primary tumor size, number of involved SNs, ECE, AJCC classification, diameter, and penetrative depth of the

TABLE 3. Comparison of categorical clinicopathological and $S N$ characteristics in invasive breast cancer patients without and with non-SN metastases by Pearson chi-square test

\begin{tabular}{|c|c|c|c|}
\hline & $\begin{array}{l}\text { Without non-SN } \\
\text { metastases }\end{array}$ & $\begin{array}{l}\text { With non-SN } \\
\text { metastases }\end{array}$ & $P$ value \\
\hline Feature & No $(\%)$ & No $(\%)$ & \\
\hline Total & $221(62)$ & $136(38)$ & \\
\hline Histological subtype & & & 0.914 \\
\hline Ductal & $183(51)$ & $115(32)$ & \\
\hline Lobular & $22(6)$ & $14(4)$ & \\
\hline Others & $16(5)$ & $7(2)$ & \\
\hline Histological grade (B\&R) & & & 0.798 \\
\hline ER positive & $198(56)$ & $124(35)$ & 0.715 \\
\hline Negative & $22(6)$ & $12(3)$ & \\
\hline PR positive & $179(50)$ & $106(30)$ & 0.589 \\
\hline Negative & $41(12)$ & $29(8)$ & \\
\hline HER-2/neu positive & $16(9)$ & $10(6)$ & 0.670 \\
\hline Negative & $83(47)$ & $67(38)$ & \\
\hline No. of SN & & & 0.246 \\
\hline 1 & $131(37)$ & $89(25)$ & \\
\hline$>1$ & $90(25)$ & $47(13)$ & \\
\hline No. of involved SN & & & 0.050 \\
\hline 1 & $198(55)$ & $112(31)$ & \\
\hline$>1$ & $23(6)$ & $24(7)$ & \\
\hline AJCC SN metastases & & & $<0.001$ \\
\hline ITC & $21(6)$ & $3(1)$ & \\
\hline Micrometastasis & $86(24)$ & $26(7)$ & \\
\hline Macrometastasis & $114(32)$ & $107(30)$ & \\
\hline Extracapsular extension & & & $<0.001$ \\
\hline No & $175(49)$ & $82(23)$ & \\
\hline Yes & $46(13)$ & $54(15)$ & \\
\hline $\begin{array}{l}\text { Microanatomic } \\
\text { location SN metastases }\end{array}$ & & & $<0.001$ \\
\hline Subcapsular & $126(35)$ & $41(11)$ & \\
\hline Combined & $23(6)$ & $17(5)$ & \\
\hline Parenchymal & $8(2)$ & $3(1)$ & \\
\hline Extensive & $64(18)$ & $75(21)$ & \\
\hline Multifocality & & & 0.087 \\
\hline No & $176(49)$ & $118(33)$ & \\
\hline Yes & $45(13)$ & $18(5)$ & \\
\hline
\end{tabular}

TABLE 4. Comparison of continuous clinicopathological and $S N$ characteristics in invasive breast cancer patients without and with non-SN metastases by Mann-Whitney U-

test

\begin{tabular}{lllr}
\hline & $\begin{array}{l}\text { Without non-SN } \\
\text { metastases }\end{array}$ & $\begin{array}{l}\text { With non-SN } \\
\text { metastases }\end{array}$ & $P$ value \\
\hline Feature & & & \\
$\begin{array}{l}\text { Total no. (\%) } \\
\text { Age (years, mean) }\end{array}$ & $221(62)$ & $136(38)$ & \\
$\begin{array}{c}\text { Primary tumor } \\
\quad \text { diameter, cm (mean) }\end{array}$ & 2.3 & 53 & 0.249 \\
$\begin{array}{c}\text { MAI, 2mm (mean) } \\
\text { SN tumor diameter, }\end{array}$ & 13 & 2.5 & 0.019 \\
$\quad$ mm (mean) & 3.6 & 12 & 0.693 \\
$\begin{array}{c}\text { SN tumor penetrative } \\
\quad \text { depth, mm (mean) }\end{array}$ & 2.2 & 6.5 & $<0.001$ \\
\hline
\end{tabular}


SN metastatic deposit (Tables 3 and 4). None of the other classic variables (age, histological subtype and grade, steroid receptor and HER-2/neu status, MAI) of the primary tumor correlated significantly with non-SN involvement.

The primary tumor features histological grade and diameter were associated with multiple $\mathrm{SN}$ tumor deposits $(P=0.03$ and 0.05 , respectively).

Frequency of non-SN metastases in patients with SN ITC $(N=24)$, micro- $(N=112)$ and macrometastases $(N=221)$ was $12.5 \%, 23 \%$, and $48 \%$, respectively $(P<0.001$, Fig. 3). Of those three patients with SN ITC and involved non-SNs, two had a non-SN micro- and one had a non-SN macrometastasis. One of these ITC was located in the parenchyma, the other two patients had a subcapsular location. Two of these three patients with SN ITC and non-SN involvement showed multiple small cell clusters and single cells in the SN.

The microanatomic localization of SN metastatic deposits correlated with non-SN involvement. Patients with subcapsular $(N=167)$, combined subcapsular and parenchymal $(N=40)$, parenchymal $(N=11)$ and extensive $(N=139)$ tumor deposits showed nonSN involvement in $25 \%, 42 \%, 27 \%$, and $54 \%$ of cases, respectively (Table 3). Morphometrically assessed penetrative depth of SN metastases was also associated with non-SN involvement. Frequency of non-SN metastases in patients with a $\mathrm{SN}$ tumor penetrative depth $<0.155 \mathrm{~mm} \quad(N=29), \quad 0.155-2.7 \mathrm{~mm}$ $(N=181)$ and $>2.7 \mathrm{~mm}(N=147)$ was $10 \%, 28 \%$, and $56 \%$, respectively $(P<0.001$, Table 5$)$. In ROC analysis, diameter (AUC $=0.686$ ) and the penetrative depth (AUC $=0.680$ ) of the SN tumor deposit had comparable discriminative value (Fig. 4).

In multivariate analysis, SN tumor diameter $(P=0.032)$ and $\mathrm{SN}$ tumor penetrative depth ( $<0.155 \mathrm{~mm}$ versus $0.155-2.7 \mathrm{~mm}$ versus $>2.7 \mathrm{~mm}$ ) $(P=0.015)$ were significant in predicting non-SN involvement. Cutoff values were interactively statistically as those best discriminating between low and high risk of non-SN metastases. Risk stratification by combining these features identified a low-risk group, an intermediate-risk group, and a high risk group for non-SN metastases with frequency of non-SN metastases in $11 \%, 29 \%$, and $56 \%$ of patients, respectively (Table 6). Frequencies of non-SN involvement in patients with SN micrometastases and a subcapsular $(N=91)$, combined subcapsular and parenchymal $(N=15)$, and parenchymal location $(N=6)$ were $21 \%, 40 \%$, and $17 \%$, respectively. Frequencies of non$\mathrm{SN}$ involvement in patients with SN macrometastases and a subcapsular $(N=54)$, combined subcapsular

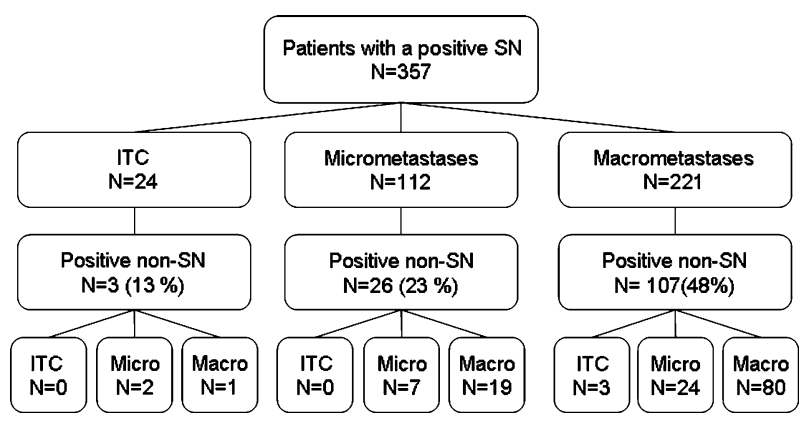

FIG. 3. Flow chart showing distribution of SN AJCC classification according to non-SN involvement.

TABLE 5. Predictive value of penetrative depth of $S N$ metastases for non-SN involvement in patients with invasive breast cancer

\begin{tabular}{lcc}
\hline $\begin{array}{l}\text { SN penetrative } \\
\text { depth (mm) }\end{array}$ & $N$ & $\begin{array}{l}\text { No. of patients with } \\
\text { non-SN involvement }(\%)\end{array}$ \\
\hline$<0.155$ & 29 & $3(10)$ \\
$0.155-2.7$ & 181 & $51(28)$ \\
$>2.7$ & 147 & $82(56)$ \\
\hline
\end{tabular}

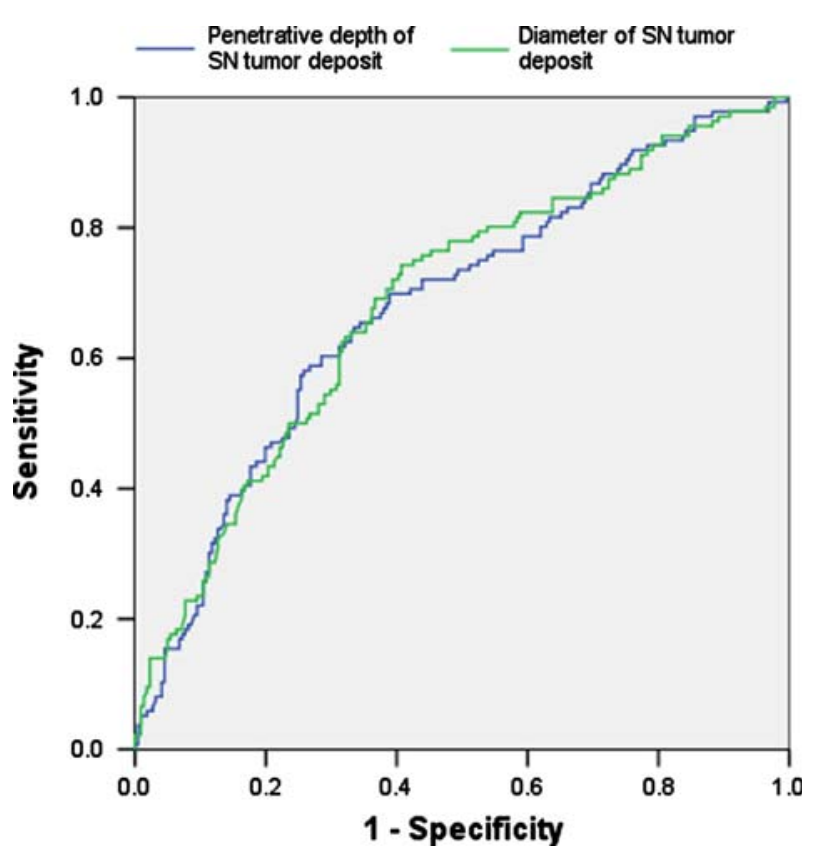

FIG. 4. ROC curves showing the sensitivity and specificity of SN tumor diameter and penetrative depth as predictors of non-SN involvement. The larger the area below the curve, the more accurate the prediction of non-SN involvement $(P<0.001)$.

and parenchymal $(N=25)$, parenchymal $(N=4)$ and extensive location $(N=138)$ were $39 \%, 44 \%$, $25 \%$, and $54 \%$, respectively. 
TABLE 6. Predictive value of a combination of AJCC classification of SN metastases and penetrative depth of SN metastases for non-SN involvement in patients with invasive breast cancer

\begin{tabular}{lcc}
\hline & $N$ & No. of patients with non-SN involvement (\%) \\
\hline ITC/SN penetrative depth $<2.7 \mathrm{~mm}$ or & 38 & $4(11)$ \\
$\quad$ Micrometastases/penetrative depth $<0.155 \mathrm{~mm}$ & 172 & $50(29)$ \\
Micro- or macrometastases/penetrative depth $0.155-2.7 \mathrm{~mm}$ & 147 & $82(56)$ \\
Micro- or macrometastases/penetrative depth $>2.7 \mathrm{~mm}$ & & \\
\hline
\end{tabular}

\section{DISCUSSION}

The lymphatic spread of breast cancer cells has been shown to follow an orderly progression via the $\mathrm{SN}$ to non-SNs, which implies that the risk of spread of tumor from the SN to the non-SN may depend on the extent of SN involvement. While many studies have focused on the maximal diameter of the metastatic tumor deposit ${ }^{22,} 23$ we investigated the microanatomic location and the penetrative depth of the metastatic deposits as a putative predictor of non-SN involvement. This was based on the concept that within the SN, tumor cells also follow an orderly route, arriving in the subcapsular sinuses through an afferent lymph vessel. Later, there is subcapsular outgrowth of malignant cells in the marginal sinuses and into the cortical parenchyma. Finally, these cells extend to the deeper zones of the lymph node parenchyma, frequently following the medullary sinuses to efferent lymph vessels. ${ }^{24}$ Consistent with this concept we found that the microanatomic location, the size, and the penetrative depth of SN tumor deposits were correlated significantly with non-SN involvement. This finding is consistent with recent melanoma studies. Startz et al. ${ }^{11}$ proposed a micromorphometric classification, based on the depth of the metastasis from the capsule and the number of 1$\mathrm{mm}$ slices containing melanoma. This classification was a highly significant predictor for distant metastases and overall survival. Dewar et al. ${ }^{13}$ also recorded that the microanatomic location of melanoma SN metastases predicts non-SN involvement, and proposed that it would be possible to safely avoid a lymph node dissection in patients with subcapsular deposits only. Indeed, subcapsular location and small tumor penetrative depth correlated with less non-SN involvement in our breast cancer study, although no subgroup of patients could be selected without nonSN involvement.

The measurement of the SN tumor penetrative depth was difficult in many cases, especially in case of extensive tumor deposits. In these cases the tumor deposits frequently extended beyond the center of the lymph node, making it difficult to determine which edge of the SN capsule should be used to measure the tumor penetrative depth. Similar difficulties were encountered when the SN had a lobulated outline. Further studies which more rigorously define the tumor penetrative depth may strengthen its predictive power and reproducibility.

In conclusion, patients with breast carcinoma and $\mathrm{SN}$ involvement can be stratified into subgroups at significantly different risk for non-SN involvement, according to microanatomic localization of the SN metastatic deposits and penetrative depth into the $\mathrm{SN}$. However, based on these features no subgroup of patients could be selected without non-SN involvement.

\section{ACKNOWLEDGEMENTS}

Supported by the AntoniusMesosGroup, Oncology Center, Nieuwegein.

\section{OPEN ACCESS}

This article is distributed under the terms of the Creative Commons Attribution Noncommercial License which permits any noncommercial use, distribution, and reproduction in any medium, provided the original author(s) and source are credited.

\section{REFERENCES}

1. Giuliano AE, Kirgan DM, Guenther JM, et al. Lymphatic mapping and sentinel lymphadenectomy for breast cancer. Ann Surg 1994; 220:391-8; discussion 398-401.

2. Krag D, Weaver D, Ashikaga T, et al. The sentinel node in breast cancer-a multicenter validation study. $N$ Engl $J$ Med 1998; 339:941-6.

3. Rietman JS, Geertzen JH, Hoekstra HJ, et al. Long term treatment related upper limb morbidity and quality of life after sentinel lymph node biopsy for stage I or II breast cancer. Eur J Surg Oncol 2006; 32:148-52.

4. van Diest PJ, Torrenga H, Meijer S, et al. Pathologic analysis of sentinel lymph nodes. Semin Surg Oncol 2001; 20:238-45.

5. Cserni G, Gregori D, Merletti F, et al. Meta-analysis of nonsentinel node metastases associated with micrometastatic sentinel nodes in breast cancer. Br J Surg 2004; 91:1245-52.

6. Samoilova E, Davis JT, Hinson J, et al. Size of sentinel node tumor deposits and extent of axillary lymph node involvement: 
which breast cancer patients may benefit from less aggressive axillary dissections?. Ann Surg Oncol 2007; 14:2221-7.

7. Viale G, Maiorano E, Pruneri G, et al. Predicting the risk for additional axillary metastases in patients with breast carcinoma and positive sentinel lymph node biopsy. Ann Surg 2005; 241:319-25.

8. Turner RR, Chu KU, Qi K, et al. Pathologic features associated with nonsentinel lymph node metastases in patients with metastatic breast carcinoma in a sentinel lymph node. Cancer 2000; 89:574-81.

9. Saidi RF, Dudrick PS, Remine SG, et al. Nonsentinel lymph node status after positive sentinel lymph node biopsy in early breast cancer. Am Surg 2004; 70:101-5; discussion 105.

10. Van Zee KJ, Manasseh DM, Bevilacqua JL, et al. A nomogram for predicting the likelihood of additional nodal metastases in breast cancer patients with a positive sentinel node biopsy. Ann Surg Oncol 2003; 10:1140-51.

11. Starz H, Balda BR, Kramer KU, et al. A micromorphometrybased concept for routine classification of sentinel lymph node metastases and its clinical relevance for patients with melanoma. Cancer 2001; 91:2110-21.

12. Scolyer RA, Li LX, McCarthy SW, et al. Micromorphometric features of positive sentinel lymph nodes predict involvement of nonsentinel nodes in patients with melanoma. Am J Clin Pathol 2004; 122:532-9.

13. Dewar DJ, Newell B, Green MA, et al. The microanatomic location of metastatic melanoma in sentinel lymph nodes predicts nonsentinel lymph node involvement. J Clin Oncol 2004; 22:3345-9.

14. Reeves ME, Delgado R, Busam KJ, et al. Prediction of nonsentinel lymph node status in melanoma. Ann Surg Oncol 2003 $10: 27-31$
15. Cserni G.. Sentinel lymph-node biopsy-based prediction of further breast cancer metastases in the axilla. Eur J Surg Oncol 2001; 27:532-8.

16. van Deurzen $\mathrm{CH}$, van Hillegersberg R, Hobbelink MG, et al. Predictive value of tumor load in breast cancer sentinel lymph nodes for second echelon lymph node metastases. Cell Oncol 2007; 29:497-505.

17. van Diest PJ, Peterse HL, Borgstein PJ, et al. Pathological investigation of sentinel lymph nodes. Eur J Nucl Med 1999; 26:S43-9.

18. van Diest PJ.. Histopathological workup of sentinel lymph nodes: how much is enough?. J Clin Pathol 1999; 52:871-3.

19. de Jong JS, van Diest PJ, Baak JP. Hot spot microvessel density and the mitotic activity index are strong additional prognostic indicators in invasive breast cancer. Histopathology 2000; 36:306-12.

20. Cserni G, Bianchi S, Boecker W, et al. Improving the reproducibility of diagnosing micrometastases and isolated tumor cells. Cancer 2005; 103:358-67.

21. Ruiter DJ, Spatz A, van den Oord JJ, et al. Pathologic staging of melanoma. Semin Oncol 2002; 29:370-81.

22. Barranger E, Coutant C, Flahault A, et al. An axilla scoring system to predict non-sentinel lymph node status in breast cancer patients with sentinel lymph node involvement. Breast Cancer Res Treat 2005; 91:113-9.

23. Chu KU, Turner RR, Hansen NM, et al. Sentinel node metastasis in patients with breast carcinoma accurately predicts immunohistochemically detectable nonsentinel node metastasis. Ann Surg Oncol 1999; 6:756-61.

24. Borgstein PJ, Meijer S, Pijpers RJ, et al. Functional lymphatic anatomy for sentinel node biopsy in breast cancer: echoes from the past and the periareolar blue method. Ann Surg 2000; 232:81-9. 